Chávez E. y cols.

Bev. Chil. Pedintr. 62 (4); 238-241, 1991

\title{
Enfermedad celíaca en la adolescencia
}

\author{
Eduardo Chávez C. ${ }^{1}$; Sergio Ceresa $\mathrm{O}^{1}$; Gladys Guevara $P .{ }^{1}$; Julio Espinoza $\mathbf{M}^{2}$; \\ Bárbara Pizarro M. $^{1}$; Maureen Rossel G.'
}

Health status in adolescents with celiac disease

\begin{abstract}
Nutritional status, sexual maturation and intestinal absorotion were assessed in 16 adolescent celiac patients (12 females) at ages 12 and 18 year. Weight/age, height/age and weight/height were $75.5 \%$ (51.6-94.8), 90.3\% (76.8$104.6)$ and $99.1 \%(76.3-112.9)$ at 12 years and $85.6 \%\{64.0-105.2\}, 93.8 \%(85.9-701.2)$ and $110 \%(76.5-121.9\}$ at 18 years of age, respectively. Sexual maturation (Tanner) was at stage I in 31\%, and at stage I1 in $43.7 \%$ of partents at age 12 years and it had reached maturity in $75 \%$ of them at 18 years, Menarchy occurred between 12 and 14 years of age, except in 3 patients in wham it was retarded. Mean serum carotene levels were 125 and $108.5 \mathrm{ug} / \mathrm{dl}$ at ages 12 and 18 years respectively. Delayed weight and height progress in this particular group of patients might be explained by low socioeconomic conditions, late dlagnosis, and poor adherence to gluten-free diet.

(Key wards: celiac disease, giuten tree diet, nutrition, sexual development, intestinal absorption.)
\end{abstract}

La enfermedad celíaca es una de las causas frecuentes de diarrea crónica y retardo del crecimiento durante la edad pediátrica. Se caracteriza por mala absorción intestinal, retardo del crecimiento y lesiones características de la mucosa del intestino delgado ${ }^{1-3}$. Se han hecho pocas descripciones nacionales sobre esta enfermedad durante la adolescencia. Hemos percibido que los pacientes celíacos y sus familias cumplen adecuadamente con su seguimiento periódico y con las indicaciones dietéticas especificas antes de la adolescencia, pero al llegar a ella se producen inasistencias reiteradas a los controles e inadecuado cumplimiento del rếgimen dietético, que pudietan guardar telación con el bienestar relativo que produce la disminución de los síntomas que ocurre a esta edad ${ }^{4}$ y a ciertas reacciones características de la personalidad de los adolescentes como rebeldfa o independencia.

El propósito de este estudio fue describir y correlacionar el desarrollo puberal, el estado nutricional y algunos parámetros de absorción intestinal en un grupo de enfermos celíacos adolescentes controlados periódicamente.

1. Unidad de Gastroenterología Infantil, Servicio de Pediatr ía, Hospital Clínico Sar Borja-Atriarán.

2. Unidad de Gastroenterología Infantil, INTA, Universidad de Chile.

\section{Pacientes y procedimientos}

En la policlinica de gastroenterología pediátrica del Hospital San ( $\mathrm{F} \infty$.) Borja-(M) Arriarán (Ex P. Jaraquemada) se han controlado regularmente desde 1970 hasta la fecha airededor de 100 enfermos celiacos, cuyo dizgnóstico cumple con los criterios de la "European Society of Paediatric Gastroenterology and Nutrition" (ESPGAN)'. Entre ellos, se seleccionaron 16 pacientes cuyos registros clínicos permitieron hacer una evaluación clínica, nutricional, puberal $y$ de laboratorio a los 12 y a los 18 años de ad.

El estado nuticional se evaluó midiendo el peso y la talla, expresándolos como peso para ta edad (P/E), talla para la edad $(T / E)$ y peso para la talla $(P / T)$, en términos del porcentaje de adecuación al percentil 50 de las curvas del Centro de Esta dísticas de Salud de los EUA (NCHS) ${ }^{6}$, Para describir el desarrollo puberal se usaron los criterios de Tanner ${ }^{7}$, considerándose como edad promedio normal de henarquia para nuestra población los 12,6 años 8 . Como indicador de absorción intestinal se utilizó la $\infty$ ncentración plasmática de caroteno (carotinemia normal $>60 \mu \mathrm{g} / \mathrm{dl}$ ) promedio de las 6 determinaciones más cercanas a las edades elegidas para el estudio, de las obtenidas durante un período de seis meses.

\section{Resultados}

De los 16 pacientes, 12 eran del sexo femenino. E1 diagnóstico histológico se realizó en pro. medio a la edad de 5 años 1 mes (márgenes 1 año 3 meses a 12 años), en tres pacientes antes de los 2 años; en 8 entre 2 años 1 mes y 6 años; en 
5 entre 6 años 1 mes y los 12 años. En la tabla se describen las relaciones $\mathrm{P} / \mathrm{E}, \mathrm{T} / \mathrm{E}, \mathrm{P} / \mathrm{T}$ y el grado de desarrollo puberal registradas a las edades de 12 y 18 años en ellos.

A los doce años, la relación $\mathrm{P} / \mathrm{E}$ en $(12 / 16)$ pacientes $(75 \%)$ se encontraba en valores bajo $90 \%$ de adecuación al percentil 50 , y en $T / E$ y $\mathrm{P} / \mathrm{T}$ estaba bajo $95 \%$ en 13 y 4 pacientes, respectivamente. A los 18 anos el porcentaje de adecuación de la relación $\mathrm{P} / \mathrm{E}$ y $\mathrm{T} / \mathrm{E}$ era inferior a $90 \%$ en 10 pacientes $y$ bajo $95 \%$ en 3 pacientes.

El desarrollo puberal a los 12 años en $31 \%$ (5/16) correspondía a la etapa I y en $43,7 \%$ (7/16) a la etapa II. A los 18 afios $75 \%(12 / 16)$ de los sujetos habían completado su desarrollo puberal. Los otros 4 pacientes que a los 18 años estaban en la etapa IV del desarrollo puberal también habían mostrado los menores grados de desarrollo a los 12 años; además ellos tenían, en la última de las dos evaluaciones, relaciones T/E por debajo del promedio del grupo. La edad de la menarquia fluctuó entre 12 y 14 años para la mayoría de estos nifros, con excepción de tres pacientes, en que ocurrió entre los 15 y 16 años (pacientes 6,15 y 16 de la tabla). Estos últimos pacientes no presentaban, a los 12 años de edad, evidencia de desarrollo puberal y tenían relaciones $\mathrm{P} / \mathrm{E}$ y $\mathrm{T} / \mathrm{E}$ bajas.
Las carotinemias promedio a los 12 y 18 años fueron 125 y $108,5 \mu \mathrm{g} / \mathrm{dl}$, respectivamente, en los niños que estaban con régimen sin gluten. Sólo en dos casos la carotinemia estaba bajo los límites normales, en uno a los 12 años, momento en que se estaban haciendo procedimientos del diagnóstico, y en otro a los 18 años en quien se comprobó franca transgresión de la dieta.

\section{Comentario}

La edad en que se hizo el diagnóstico de enfermedad celíaca en nuestros pacientes fue muy variable y más tardía que en otras series $4,9,10$, lo que probablemente se debe a referencia tardía de nuestros casos a gastroenterología. El predominio de sexo femenino, entre los adolescentes de nuestra serie, también contrasta con otros estudios $^{\mathbf{4}, 9}$, en que ésta fue de $1,2: 1$. Esto podría ser explicado por mayor adherencia de las niñas a los controles médicos.

Las relaciones $\mathrm{P} / \mathrm{E}$ y $\mathrm{T} / \mathrm{E}$ a los 18 años mejoraron con respecto a las de 12 años, pero sin a!canzar la completa adecuación al estándar. En 2 pacientes el período de régimen sin gluten hatía sido corto, ya que el diagnóstico se hizo precisamente a los 12 años. Si bien en otra serie

Tabla

Porcentaje de adecuación al percentil 50 (NCHS) de peso para la edad (P/E),

talla para la edad (T/E), peso para la talla (P/T) y etapa del

desartollo puberal (Tanner) a los 12 y 18 años

(a) de edad en 16 adolescentes con enfermedad celíaca

\begin{tabular}{|c|c|c|c|c|c|c|c|c|}
\hline \multirow{2}{*}{$\begin{array}{l}\text { Caso/ } \\
\text { Edad }\end{array}$} & \multicolumn{2}{|c|}{$P / E(\%)$} & \multicolumn{2}{|c|}{$\mathrm{T} / \mathrm{E}\left(\boldsymbol{\psi}_{0}\right)$} & \multicolumn{2}{|c|}{$P / T(\%)$} & \multicolumn{2}{|c|}{ Desatrollo pubetal } \\
\hline & 12 & 18 & 12 & 18 & 12 & 18 & 12 & 18 \\
\hline 1 & 83,9 & 89,4 & 91,7 & 92,7 & 107,5 & 121,4 & DI & v \\
\hline 2 & 92,9 & 989 & 94,3 & 100,3 & 108,8 & 98,0 & II & V \\
\hline 3 & 68,3 & 87,7 & 87,4 & 92,0 & 100,0 & 1219 & III & $\mathrm{v}$ \\
\hline 4 & 902 & 1052 & 98,0 & 101,2 & 94,8 & 101,6 & II & V \\
\hline 5 & 719 & 77,7 & 86,7 & 90,2 & 108,2 & 113,5 & II & V \\
\hline 6 & 74,6 & 94,2 & 93,0 & 97,5 & 91.3 & 107,4 & I & $\mathrm{V}$ \\
\hline 7 & 94,8 & 83,4 & 104,6 & 98,8 & 84,3 & 89,8 & III & V \\
\hline 8 & 73,1 & 92,9 & 90,0 & 100,6 & $9 B, 3$ & 913 & II & V \\
\hline 9 & 67,0 & 789 & 82,7 & 91,1 & 97,3 & 97,8 & II & V \\
\hline 10 & 60,5 & 73,3 & 85,4 & 86,4 & 95,3 & 119,4 & II & IV \\
\hline 11 & 79,7 & 81,7 & 88,6 & 94,0 & 112,9 & 1059 & II & $\mathrm{V}$ \\
\hline 12 & 685 & 75,7 & 88,0 & 91,2 & 98,7 & 116,4 & I & IV \\
\hline 13 & 912 & 83,3 & 97,0 & 90,2 & 98,4 & 120,0 & III & $\mathrm{V}$ \\
\hline 14 & 62,5 & 64,0 & 93,3 & 96,3 & 76,3 & 76,5 & I & V \\
\hline 15 & 77,3 & 90,8 & 88,7 & 92,0 & 108,3 & 126,0 & I & IV \\
\hline 16 & 51,6 & 91,7 & 76,8 & 85,9 & 1059 & 153,0 & I & JV \\
\hline$\overline{\mathrm{X}}$ & 75,5 & 85,6 & 90,3 & 93,8 & 99,1 & 110,0 & & \\
\hline
\end{tabular}


de pacientes diagnosticados después de los 5 años de edad la recuperación del peso y la talla fueron adecuadas ${ }^{11}$. Otros no encontraron diferencias notorias de los percentiles de peso y talla entre sus pacientes y la población general, pero esos valores eran significativamente măs bajos que los de sus propios controles ${ }^{9}$. Es muy posible que existan deficiencias educacionales y socioeconómicas en la población estudiada por noso. tros que podrían explicar estos hallazgos, los que estamos intentando resolver mediante la creación de clubes de celíacos y corporaciones destinadas a ayudar a estos pacientes ${ }^{12}, 13$

El lento desarrollo puberal de nuestros pacientes guardaba relación con el estado insatisfactorio de la nutrición; sin embargo, en la mayoría de ellos se había completado a los 18 años, como podía esperarse de otras experiencias ${ }^{2}$. El retraso de la edad de la menarquia observado en algunas niñas también ha sido descrito por otros'. E1 insuficiente desarrollo pondoestatural a los $12 \mathrm{y}$ 18 años no se acompañó de carotinemias bajas, tnás aún, èstas eran normales en los niños que a $\operatorname{los} 12$ años tenían desarrollo puberal en eta. pa I de Tarnner. Si, por otra parte, el estado nutricional y el desarrollo puberal reflejan una adecuada absorción de nutrientes, estos resultados pondrían dudas sobre la utilidad de las medicio. nes de caroteno plasmático como indicador de absarción intestinal a estáa edad. Otros trabajos han señalado, sin embargo, que la medición de caroteno sérico es de alta sensibilidad y serfa útil para la detección de niños con enfermedad celíaca que transgreden el régimen ${ }^{14,}$, $y$ en nuestra experiencia (observaciones no publicadas) hay buena correlación entre las concen. traciones de caroteno sérico y la absorción intestinal en edades tempranas de la vida. El aumento de absorción por los segmentos distales - no dañados- del intestino delgado en adoles. centes celíacos podría explicar discrepancias entre absorción intestinal $y$ carotinemia en este período de la vida.

Junto con las consecuencias nutricionales y endocrinas de la enfermedad celíaca, cuyo seguimiento, por su importancia, requerirá del desarrollo y aplicación de nuevos métodos de laboratorio más precisos y fieles de diagnóstico y evaluación, como los anticuerpos antigliadina, por ejemplo ${ }^{16,17}$, conviene mencionar el riesgo que tienen estos pacientes de desarrollar tumores malignos, problema que, aun siendo poco frecuente y más propio de los adultos, también puede afectar a adolescentes, en quienes se han descrito fibrosarcomas y linfo. $\operatorname{mas}^{18,19}$ no observados en esta serie.

\section{Resumen}

Sc describen el grado de desarrollo puberal $y$ el estado nutricional en 16 pacientes celíacos adolescentes, a los 12 y 18 años de edad. La edad promedio al diagnóstico fue de 5 años 1 mes, con márgenes de 1 años 3 meses y 12 años. Los porcentajes promedios de adecuación al percentil 50 de $P / E$ fueron $75,5 \%$ y $85,6 \%$ y los de T/E 90,3 y 93,8 a a los 12 y 18 años, respectivamente. El desarrollo puberal, según la calificación de Tanner, estaba en etapa I en $31 \%$ y II en $43,7 \%$ de los casos y a los 18 años $75 \%$ de los pacientes lo había completado. $\mathrm{La}$ edad de menarquia fue la normal para nuestra población, salvo en tres pacientes. La carotine. mia promedio era 125,5 y de $108,5 \mu \mathrm{g} / \mathrm{dl}$ a los 12 y 18 años y no guardaba relación con la evolución ni el cumplimiento de la dieta. El inadecua. do desarrollo pondoestatural que alcanzaron estos niños a los 18 años probablemente dice relación con diagnóstico tardío, deficientes niveles socioeconómico y cultural de la población en estudio y la pobre adherencia al régimen sin gluten. Concluimos que es necesario procurar diagnớsticos más precoces, manejo integral y seguimientos estrictos durante la adolescencia de estos pacientes, estimulando la creación de clubes de celíacos y la dotación de nuestros la. boratorios con nuevos exámenes para el seguimiento de estos enfermos.

(Palabras claves: enfermedad celíaca, adolescentes, desarrollo, crecimiento, carotinemia.)

\section{Referencias}

1. Branski, D.: Lebenthal, E.: Celiac disease, En: Textbook of Gastroenterology and Nutrition in Infancy. E. Lebenthal, ed. Raven Press New York. 1981; 1013-1025.

2. Auricchio, $S$; Greco, L; Troncone, R.:- Glutensensitive enteropathy in childhood. Pediate Clin North Am 1988; 35: 157-187.

3. Ceresa, S.: Enfermedad celíaca, Rev Chil Pediatr 1988; 59: (Supl 2): 22-25.

4. Macki, M.; Löhdeaho, M.; Hallström, O.; Viander.

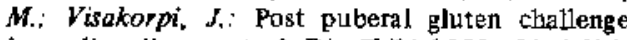
in cocliac disease. Arch Dis Child 1989; 64: 1604 1607 . 
5. Meeuwisse, $G$, Round table discussion. Diagnostic criteria for celiac disease. Acta Paediatr Scand 1970; 59: 461-463.

6. National Center for Heaith Statistics: NCHS growth charts. Monthy Vital Statistics Report, Rockeville, Maryland, DHEW publication $\mathrm{N}^{\circ}$ (H.R.A.). 1976; 25 (Suppl 3): 76-1120.

7. Tonner, J.M.: Growth at adolescence, 2nd Edition. Oxford: Blackwell Scientific Publications, 1962.

8. Cattani, $A$.: pubertad precoz y retardada. Rev Chil Pediatr 1988;59: (Supl 3): 13-19

9. Kumar, P.; Walker-Smith, J.; Milla, P.; Harris, G., Colyer, J.: Hallidey, $R$.: The teenage Coeliac: follow up study of 102 patients. Arch Dis Child $1988 ; 63: 916-920$.

10. Guiraldes. E,; Gutiérrez, C; Bancalari, A.; Campbell, $M$; Kunzagk, $H$, Vargas, $A_{\text {.: }}$ Causas de diamea crónica infantil. Rev Chil Pediatr 1985; 56 : 418 421 .

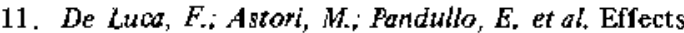
of a gluten-fiee diet on catch up growth and height prognosis in celias children with growth retardation recognized after the age of 5 years. Eur J Pediatr 1988; 147: 188-191.

12. Espinoza, J.; Rossel, M.; Ceresa, S.: Araya, M.; Atak, M.E.: Club de celjacos, una alternativa para el tratamiento integral de los enfermos celíacos. Rev Chil Pediatr 1985; 56: 468-472.
13. Rossel, M; Araya, M.: Corporación de Ayuda al Celíaco COACEL. (Carta al Editor). Rev Chil Pediatr 1990; 61: 117-118.

14. Naveh, Y.; Ken-Dror, A.; Zinder, O.; Berkovich, D.: Comparatjve reliability of D-xilose absorption and serum B-carotene measurements in small intestinal disease, J Pediatr Gastroenterol Nutr 1986; 5: 210 213.

15. Guiraldes, E.; Gutiërrez, C,; Rebolledo, L, harin, $P$.: El examen del caroteno sérico en la diarrea crónica de la infancia. Rev Chil Pediatr 1974; 45: $409-415$

16. Bürgin-Walff, A,; Bertele, R.; Bergeur, et al.: A reliable screening test for childhood celiac disease: Fluorescent immunosorbent test of gliadin antibodies. J Pediatr 1983; 102: 650-660.

17. O'Farrell, L., Kelly, J., Hekkens, W. et al.: Gliadin antibody levels: A serological test for coeliac disease. Bu Med J 1983; 286: 2007-2010.

18. Amaud-Bathandier, $F_{.:}$Schmitz, $J_{. i}$ Ricour, $C_{.,}$ Rey, $J$. Intestinal malignant lymphoma in a child with familial celiac disease. J Pediatr Gastroenterol Nutr 1983; 2: 320-323.

19. Verkasalo, M.; Savilahti, E.; Rapola, J.; Wolgren, E.: Fibrosurcoma in a girl with celiac disease and Ig A deficiency. J Pediatr Gastroenterol Nutr 1985; 4 : 839-841.

\section{AVISO A LOS AUTORES}

Con el objeto de dar prioridad a los trabajos de investigacion, en vista de las limitaciones de espacio de la Revista Chilena de Pedjatría, el Comité Editorial ha acordado restringir la impresión de casos clínicos a un máximo de dos por cada número. 